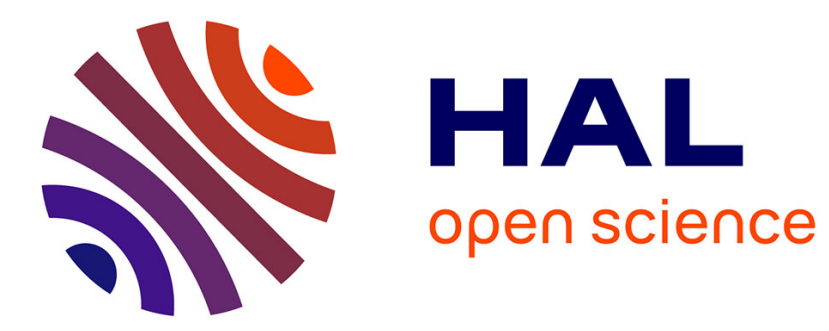

\title{
Light beating spectroscopy measurements of microemulsion diffusion coefficient
}

Alain Graciaa, Jean Lachaise, P. Chabrat, L. Letamendia, J. Rouch, C. Vaucamps, M. Bourrel, C. Chambu

\section{- To cite this version:}

Alain Graciaa, Jean Lachaise, P. Chabrat, L. Letamendia, J. Rouch, et al.. Light beating spectroscopy measurements of microemulsion diffusion coefficient. Journal de Physique Lettres, 1977, 38 (13), pp.253-257. 10.1051/jphyslet:019770038013025300 . jpa-00231371

HAL Id: jpa-00231371

https://hal.science/jpa-00231371

Submitted on 1 Jan 1977

HAL is a multi-disciplinary open access archive for the deposit and dissemination of scientific research documents, whether they are published or not. The documents may come from teaching and research institutions in France or abroad, or from public or private research centers.
L'archive ouverte pluridisciplinaire HAL, est destinée au dépôt et à la diffusion de documents scientifiques de niveau recherche, publiés ou non, émanant des établissements d'enseignement et de recherche français ou étrangers, des laboratoires publics ou privés. 


\title{
LIGHT BEATING SPECTROSCOPY MEASUREMENTS OF MICROEMULSION DIFFUSION COEFFICIENT
}

\author{
A. GRACIAA, J. LACHAISE \\ Laboratoire de Thermodynamique des Etats Métastables, \\ Institut Universitaire de Recherche Scientifique, 64010 Pau, France \\ P. CHABRAT, L. LETAMENDIA, J. ROUCH, C. VAUCAMPS \\ Laboratoire d'Optique Moléculaire, E.R. 134 du C.N.R.S. \\ Université de Bordeaux I, 33405 Talence, France \\ M. BOURREL and C. CHAMBU \\ Service de Physique des Procédés, C.R.L., SNEA (P) \\ 64170 Lacq, France
}

(Reçu le 24 mars 1977, accepté le 1 er juin 1977)

\begin{abstract}
Résumé. - On a mesuré par la méthode des battements lumineux le coefficient de diffusion de micelles formées au sein de mélanges quaternaires eau-alcool-tensioactif-toluène, pour différentes valeurs de la fraction volumique de la phase dispersée. Les résultats obtenus conduisent d'une part à admettre une monodispersion du diamètre des micelles, d'autre part à un bon accord - expliqué théoriquement - avec les mesures de turbidité.

Abstract. - The translational diffusion coefficient of microemulsions formed from water, toluol, sodium dodecyl-sulfate and alcohol, has been measured using light beating spectroscopy. The experimental results show that the microemulsions are monodisperse, the micelles being electrically charged.
\end{abstract}

1. Introduction. - Optically transparent microemulsions can be formed from water-oil-soap-alcohol mixtures. Bowcott and Schulman [1] showed the part played by alcohol as a stabilizing agent and postulated that these microemulsions were formed by an oilalcohol continuous phase, the dispersed phase being composed of water-alcohol spherical droplets protected by a soap-alcohol interface. In some conditions an inversion can arise, water becoming a constituent of the continuous phase and the oil becoming droplets. These systems can be diluted without breaking, by adding a mixture of definite composition which is actually that of the continuous phase.

With these assumptions, microemulsions behave like macromolecular solutions. Graciaa et al. [2] performed light scattering intensity and turbidity measurements on water-toluol (or benzol) -sodium dodecyl sulfate-butanol microemulsions at different volume fractions $v$ of the dispersed phase. Extrapolation of their measurements to $v \rightarrow 0$ allowed them to deduce the volume $V_{0}$ of the droplets.
We report in this paper measurements of the scattered light autocorrelation function, using light beating spectroscopy. This technique, which has been applied to micellar solutions of soap in water [3], gives the diffusion coefficient of the aggregates constituting the dispersed phase.

2. Experimental. - The experimental set-up is depicted on figure 1. A single mode-stabilized argon laser beam is split into two parts : the first one being focused in the sample, the other one, much lower in intensity, being used both as alignment beam and as a local oscillator in heterodyne light beating. An arrangement of mirrors allows us to vary the scattering angle $\theta$ and then to study fluctuations of different wave vectors $\mathbf{q}$ :

$$
\begin{aligned}
\mathbf{q} & = \pm\left(\mathbf{K}_{\mathbf{i}}-\mathbf{K}_{\mathrm{d}}\right) \\
|\mathbf{q}| & =\frac{4 \pi n}{\lambda_{0}} \sin \theta / 2
\end{aligned}
$$




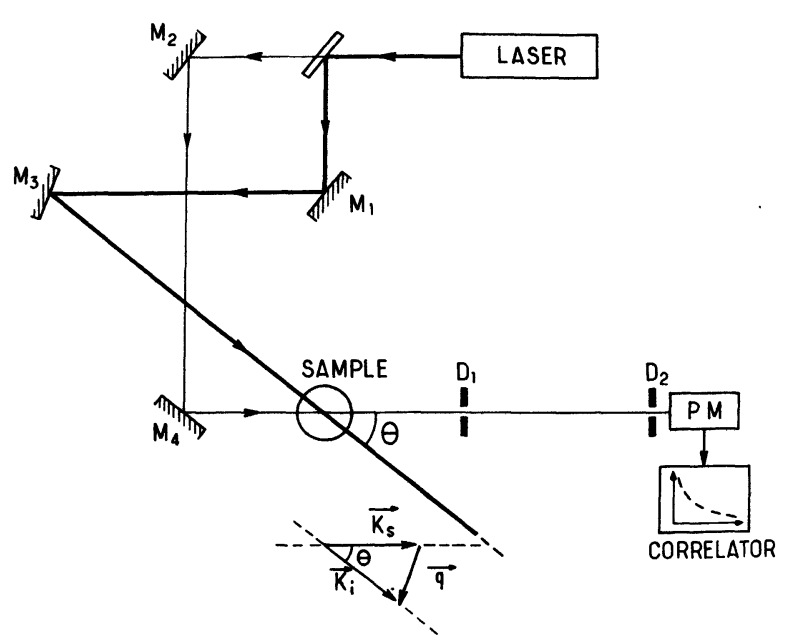

Fig. 1. - Experimental setup.

where $K_{i}$ and $K_{d}$ are the wave vectors of the laser light and scattered light respectively and $n$ is the refractive index of the sample for the laser wave length $\lambda_{0}$.

The pinholes $D_{1}$ and $D_{2}$ isolate a single coherence area in order to obtain a maximum signal/noise ratio. The pinhole $D_{2}$ is located in front of the cathode of a low dark current phototube EMI $6256 \mathrm{~S}$. The photocurrent supplies an intensity correlator which calculates the photocurrent autocorrelation function $F_{2}(t)$ at different delay times $t$

$$
F_{2}(t)=\left\langle i\left(t_{0}\right) i\left(t_{0}+t\right)\right\rangle .
$$

The brackets denote a time averaging over the initial time $t_{0}$. According to the experimental conditions either digital (an apparatus built in our laboratory from S. H. Chen's design [4] ; Malvern Correlator $\left({ }^{1}\right)$ ) or analogic (Saicor) correlators.

Both homodyne and heterodyne techniques have been used.

Measurements have been performed on watertoluol-sodium dodecylsulfate-butanol 1 microemulsions. The range covered was from 0.03 to 0.6 for the volume fraction $v$ of the dispersed phase and from $4^{\circ}$ to $130^{\circ}$ for the scattering angle $\theta$

$$
\left(1.4 \times 10^{4} \mathrm{~cm}^{-1} \leqslant q \leqslant 3.6 \times 10^{5} \mathrm{~cm}^{-1}\right) .
$$

a) The photocurrent correlation functions $F_{2}(t)$ were always satisfactorily fitted to single exponential functions. The time constant $\tau$ was deduced with an accuracy of $2-5 \%$. The relation $\tau_{2}=\tau_{1} / 2$ was obtained from heterodyning $\left(\tau_{1}\right)$ and homodyning $\left(\tau_{2}\right)$ in the same conditions. For instance, a given mixture at $\theta=35.05^{\circ}$ provides $\tau_{1}=0.543 \mathrm{~ms}, \tau_{2}=0.268 \mathrm{~ms}$.

b) A linear relation has been found between $\tau$ and $q^{-2}$ (Fig. 2) :

$$
\frac{1}{\tau_{1}}=\frac{1}{2 \tau_{2}}=D q^{2}
$$

( $\left.{ }^{1}\right)$ The authors are very grateful to Mr. Woodley from SITAF Co. for the loan of the Malvern Correlator.

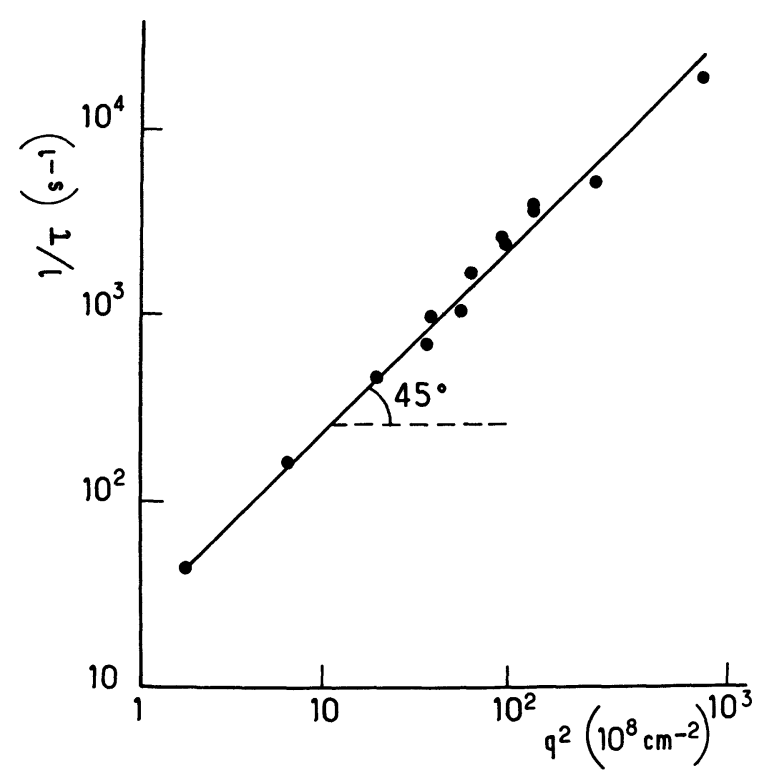

FIG. 2. - Log-Log plot of $1 / \tau$ versus $q^{2}$. The $\pi / 4$ slope shows that $1 / \tau \propto q^{2}$.

c) Electrophoresis experiments (light scattering under homogeneous continuous electric field) give an oscillating correlation function. This result shows that the droplets behave like electrically charged particles [5]. But it has not been possible to obtain a precise value of the mobility, presumably because there is too great a dispersion in the magnitude of the charges.

d) Highly turbid samples show a slight but significant depolarization of the scattered light. For $90^{\circ}$ scattering, the coherence time of the depolarized component has been found to be very close to that of the corresponding isotropic scattered light. We believe that depolarization is a result of multiple scattering, in analogy with the phenomena widely observed in critical fluids [6].

3. Discussion. - We attempt to give a consistent explanation of our light scattering data on the basis of the spherical droplet model of the microemulsion.

$N$ identical point particles, labelled from their position $\mathbf{r}_{1}(t), \ldots, \mathbf{r}_{N}(t)$, scatter the electric field $E_{\mathrm{S}}(\mathbf{q}, t)$ in the direction $\theta$ corresponding to the wave vector $\mathbf{q}$ :

$$
E_{\mathrm{S}}(\mathbf{q}, t) \sim \sum_{j=1}^{N} \exp i \mathbf{q} \cdot \mathbf{r}_{j}(t)
$$

Let $\delta \rho(\mathbf{r}, t)$ be the instantaneous fluctuation of the droplet density at the point $\mathbf{r}$ and time $t$ :

$$
\delta \rho(\mathbf{r}, t)=\sum_{j=1}^{N} \delta\left(\mathbf{r}-\mathbf{r}_{j}(t)\right)-\frac{N}{V}
$$

where $\delta(\ldots)$ symbolizes the Dirac function and $V$ is the volume containing the $N$ scatterers. 
One can easily show that the scattered field $E_{\mathrm{S}}(\mathbf{q}, t)$ is proportional to the spatial Fourier transform $\delta \rho(\mathbf{q}, t)$ of $\delta \rho(\mathbf{r}, t)$ :

$$
\delta \rho(\mathbf{q}, t) \sim \sum_{j=1}^{N} \exp i \mathbf{q} \cdot \mathbf{r}_{j}(t)
$$

Then the scattered field and scattered intensity correlation functions, $G_{1}(\mathbf{q}, t)$ and $G_{2}(\mathbf{q}, t)$ respectively, may be written :

$$
\begin{aligned}
& G_{1}(\mathbf{q}, t) \sim\langle\delta \rho(\mathbf{q}, t) \delta \rho(-\mathbf{q}, 0)\rangle \\
& G_{2}(\mathbf{q}, t) \sim\left\langle|\delta \rho(\mathbf{q}, t)|^{2} \cdot|\delta \rho(\mathbf{q}, 0)|^{2}\right\rangle .
\end{aligned}
$$

Heterodyne and homodyne techniques provide measurements of $G_{1}$ and $G_{2}$ [7] respectively.

3.1 LOW VOLUME FRACTIONS $(v \rightarrow 0)$ OF THE DISPERSED PHASE. - At any concentration, $G_{1}$ is written :

$$
G_{1}(\mathbf{q}, t) \sim\left\langle\sum_{j, l=1}^{N} \exp i \mathbf{q} \cdot\left(\mathbf{r}_{j}(0)-\mathbf{r}_{l}(t)\right)\right\rangle .
$$

At low particle concentration $(v \rightarrow 0)$ the scatterers are assumed to be statistically independent. Then, all the pair correlation terms $l \neq j$ fall out and

$$
G_{1}(\mathbf{q}, t) \sim N g_{\mathbf{s}}(\mathbf{q}, t)
$$

where $g_{\mathrm{S}}(\mathbf{q}, t)$ is the Fourier transform of the self part of the Van Hove correlation function $g_{\mathrm{S}}(\mathbf{r}, t)$. For Brownian particles, $g_{\mathrm{S}}(\mathbf{r}, t)$ obeys a diffusion equation [5]. The spatial Fourier transform of this equation is :

$$
\frac{\partial g_{\mathrm{S}}(\mathbf{q}, t)}{\partial t}=-D_{0} q^{2} g_{\mathrm{s}}(\mathbf{q}, t)
$$

where $D_{0}$ is the Stokes-Einstein diffusion coefficient :

$$
D_{0}=\frac{k_{\mathrm{B}} T}{6 \pi a \eta}
$$

$k_{\mathrm{B}}, T, a$ and $\eta$ are respectively the Boltzmann constant, the absolute temperature, the particle hydrodynamic radius and the viscosity of the continuous phase.

The model described by eq. (10) predicts exponential correlation functions $G_{1}$ and $G_{2}$, with correlation times $\tau_{1}$ and $\tau_{2}$ :

$$
\tau_{1}=2 \tau_{2}=\frac{1}{D_{0} q^{2}}=\frac{6 \pi a \eta}{k_{\mathrm{B}} T q^{2}} .
$$

Figure 3 gives a plot of the coefficient $D=1 / \tau_{1} q^{2}$ versus the volume fraction $v$ of the dispersed phase. for the scattering angle $\theta=31^{\circ} 20^{\prime}$

$$
\left(q^{2}=1.21 \times 10^{10} \mathrm{~cm}^{-2}\right) .
$$

Extrapolation to $v=0$ leads to the Stokes-Einstein diffusion coefficient $D_{0}$. For this sample

$$
D_{0}=4.75 \times 10^{-7} \mathrm{~cm}^{2} \mathrm{~s}^{-1}
$$

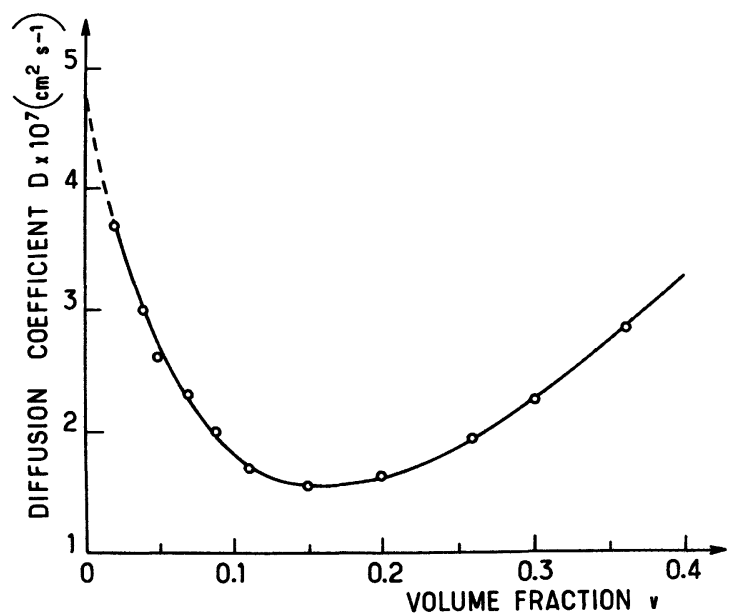

Fig. 3. - Diffusion coefficient as a function of the volume fraction of the sample.

the deduced value of the hydrodynamic radius of the droplets $a$ is

$$
a=(102 \pm 5) \AA .
$$

This result is in good agreement with the value $95 \AA$ obtained from turbidity measurements [2].

A polydispersity of the size of the droplets would lead to a non-exponential correlation function $G_{1}(\mathbf{q}, t)$ :

$$
\begin{aligned}
G_{1}(\mathbf{q}, t) \sim & \int \mathrm{d} D \varphi(D) \exp \left(-D q^{2} t\right)= \\
& =\exp \left(-\bar{D} q^{2} t\right)\left[1+\frac{\mu}{2} q^{4} t^{2}+\cdots\right]
\end{aligned}
$$

where $\varphi(D)$ is the distribution function of the diffusion coefficients, in connection with the distribution of the droplet hydrodynamic radius $a ; \bar{D}$ is the mean value, $\mu=\overline{(D-\bar{D})^{2}}$ the variance.

The correlation functions have been accurately fitted to exponentials, the correlation time of which is found proportional to $q^{-2}$. This fact allows us to conclude that the microemulsions studied are monodisperse. Taking into account the experimental errors, the degree of polydispersity [8] which is given by $\mu /(\bar{D})^{2}$ is less than $5 \%$.

3.2 HIGHER VOLUME FRACTIONS OF THE DISPERSED PHASE. - At higher particle concentrations, statistical independency of the scatterers can no longer be assumed. The distinct term $(l \neq j)$ of $G_{1}$ cannot be ignored :

$$
G_{1}(\mathbf{q}, t) \sim N g_{\mathrm{S}}(\mathbf{q}, t)+\sum_{j \neq l} \exp i \mathbf{q} \cdot\left[\mathbf{r}_{j}(0)-\mathbf{r}_{l}(t)\right] .
$$

Pusey has shown [8] that with some approximations (the Vineyard approach) the distinct part of $G_{1}$ has the same time dependence as the self part. Then $G_{1}$ and $G_{2}$ are still exponentials and their correlation 
time is connected with the diffusion coefficient $D_{\mathrm{S}}$ of a single particle in the presence of interactions.

But in order to compare turbidity and light beating data at higher volume fractions $v$, we used a hydrodynamic-thermodynamic model. For $v \geqslant 0.1$ the mean droplet distance does not differ significantly from the droplet diameter and the smallness of the product $q . a$ allows us to use this approach. The microemulsion is then considered as a non-reacting macromolecular solution. The continuous phase is the solvent and the aggregates of the dispersed phase which constitutes the solute are first assumed to be constant in size whatever the volume fraction $v$. The isothermal, isobaric fluctuations $\delta \rho(\mathbf{r}, t)$ of the droplet density are analogous to the solute concentration fluctuations in a binary mixture.

The Rayleigh constant $R$ and the turbidity $\gamma$ are proportional to the mean intensity $\langle I\rangle$ scattered by the unit scattering volume (due to the droplets and not to the continuous phase). $\langle I\rangle$ is given by :

$$
\langle I\rangle \sim n^{2}\left[\left(\frac{\partial n}{\partial c}\right)_{\mathrm{P}, \mathrm{T}}\right]^{2}\left\langle(\delta c)^{2}\right\rangle
$$

where $c$ is the concentration of the solute $\left(c=\rho / \mathcal{N}_{0}\right.$; $\mathcal{N}_{0}$ is the Avogadro number). $\langle I\rangle$ may be expressed in terms of the volume fraction $v$ of the solute and the derivative of the osmotic pressure $\Pi[9]$ :

$$
\langle I\rangle \sim v \frac{k_{\mathrm{B}} T}{\left(\frac{\partial \Pi}{\partial v}\right)_{\mathrm{P}, \mathrm{T}}} .
$$

Graciaa et al. fitted their experimental values of $R$ and $\gamma$ to the above expression [2] ; using the virial expansion of the osmotic pressure, they were able to deduce the volume $V_{0}$ of the droplets.

The dynamics of the density-fluctuations $\delta \rho(\mathbf{r}, t)$ or equivalently the concentration-fluctuations $\delta c(\mathbf{r}, t)$, is governed by a diffusion equation :

$$
\frac{\partial \delta c(\mathbf{r}, t)}{\partial t}=D_{\mathrm{S}} \nabla^{2} \delta c(\mathbf{r}, t)
$$

It is possible to calculate $D_{\mathrm{s}}$ as a function of thermodynamic properties of the solution [5]. If the osmotic pressure is used instead of the chemical potential of the solute, the expression given in [5] becomes :

$$
D_{\mathrm{S}}=\frac{1}{\mathcal{N}_{0} f}\left(\frac{\partial \Pi}{\partial c}\right)_{\mathbf{P}, \mathbf{T}}
$$

$f$ is a friction coefficient which depends on $c$.

For the ideal solution $(c \rightarrow 0)(\partial \Pi / \partial c)_{\mathrm{P}, \mathrm{T}}$ and $D_{\mathrm{S}}$ have the limit values $R T$ and $D_{0}=k_{\mathrm{B}} T / 6 \pi a \eta$ respectively, thus $f$ must have the limit $6 \pi a \eta$.

$D_{\mathrm{s}}$ may be expanded as a power series of $v$, as may $v \mid\langle I\rangle$, but with distinct coefficients due to the $v$ dependence of the friction coefficient :

$$
\begin{gathered}
\frac{v}{\langle I\rangle} \sim \frac{1}{V_{0}}\left(1+A v+B v^{2}+\cdots\right) \\
D_{\mathrm{S}}=D_{0}\left(1+A^{\prime} v+B^{\prime} v^{2}+\cdots\right) .
\end{gathered}
$$

Experimental values of $v /\langle I\rangle$ as well as $D_{\mathrm{S}}$ show large negative coefficients $A$ and $A^{\prime}\left(A^{\prime} \simeq-13\right.$ for the results plotted in figure $3 ; A \simeq--10$ for the corresponding turbidity data figure 4 ). These results have to be associated with a negative second virial coefficient of the osmotic pressure.

For rigid spherical particles, Tanford [10] calculated the second virial coefficient which equals the excluded volume, leading to $A \equiv K_{t}=8$. Theoretical treatments applied to the same particles yield

$$
A^{\prime} \equiv K_{\mathrm{D}} \simeq 1 \quad[5] \text {. }
$$

Measured sedimentation and diffusion coefficients of macromolecules such as DNA [11] agree with these theoretical values. However Pusey et al. [8] found positive and negative as well as zero values of $K_{\mathrm{D}}$ in solutions of $\mathrm{R}-17$ viruses, depending on $\mathrm{NaCl}$ concentration. Negative values have been connected with the fact that the particles become electrically charged as the $\mathrm{NaCl}$ concentration increases.

Recently Corti and Degiorgio [12] studied nonionic detergent-water micellar mixtures, their results were fitted fairly well to an alternative model in which the system is regarded as a critical mixture and no longer as well-defined particles. However no departure to the linear dependence on $q^{-2}$ of the correlation time has been observed in our case ; furthermore the scattered intensity has not been found $q$ dependent. This shows that the correlation length should be much smaller than $q^{-1}$.

There is no satisfactory explanation for the anomalous variation of $D_{\mathrm{s}}$ and $v /\langle I\rangle$ versus $v$. A better knowledge of the structure of the droplets would be very useful for estimating the different contributions in the particle-particle and particle-solvent interactions.

4. Conclusion. - We have measured, using light beating spectroscopy, the diffusion coefficient of microemulsions as a function of the volume fraction of the dispersed phase $v$. The particle hydrodynamic radius which is obtained from the diffusion coefficient extrapolated to $v=0$ is in good agreement with the turbidity and scattered intensity data on the basis of Schulman's droplet model. The exponential shape of the correlation functions is associated with a welldefined size of the aggregates (less than $5 \%$ of dispersion). The strong decrease of $D_{\mathrm{S}}$ as well as $v /\langle I\rangle$ when the volume fraction $v$ increases must be associated with a negative second virial coefficient of the osmotic pressure in the droplet model; but it is not possible to explain that property physically. 


\section{References}

[1] Bowcott, J. E., Schulman, J. H., Z. Elektrochem. 59 (1955) 283.

[2] Graciaa, A., Lachaise, J., Martinez, A., Bourrel, M., Chambu, C., C.R. Hebd. Séan. Acad. Sci. B 282 (1976) 547.

[3] Mazer, N. A., BenedeK, G. B., Carey, M. C., J. Phys. Chem. 80 (1976) 1075.

[4] Chen, S. H., VeldkampF, W., J. Sci. Instrum. 46 (1975) 1356.

[5] Berne, B. J. and PeCora, R., Dynamic Light Scattering (Wiley) 1976.

[6] BerTolotTI, M., Multiple Scattering, to be published in Proceedings of NATO Summer School on Photon Correlation and Velocimetry. Capri 1976.
[7] Pike, E. R. and Jakeman, E., Photon Statistics and Photon Correlation Spectroscopy. Adv. Quantum Electron. 2 (Academic Press) 1973.

[8] Pusey, P. N., in Photon correlation and light beating spectroscopy, Cummins, H. Z., Pike, E. R., Ed. (Plenum Press) 1974.

[9] Stacey, K. A., Light Scattering in Physical Chemistry (Butterworths) 1956.

[10] TANFORD, C., Physical Chemistry of Macromolecules (Wiley) 1961.

[11] Newman, J., Swinney, H. L., Berkowitz, S. A., Day, L. A., Biochemistry 13 (1974) 4832.

[12] Corti, M., Degiorgio, V., Opt. Commun. 14 (1975) 358. 